\title{
Mathematical modeling of rigid bodies motion through some non-Newtonian flows
}

\author{
J. Ferchichi and J. P. Zolésio
}

\begin{abstract}
The main novelty of this article is to reveal a weak formulation of rigid bodies motion arising from free boundaries evolution in viscoplastic flows governed by the incompressible Norton-Hoff operator with non-cylindrical coefficients. We supply the existence result of an interface between the two non-miscible fluids by the use of the non-smooth evolution theory. We prove that the fluid's flow is converted to a rigid body when its viscosity is large enough. The established results are variants or extensions of the existing formulations.
\end{abstract}

Mathematics Subject Classification (2010). 32A70; 35Q35; 35Q93; 47H10; 47J35; 49J59; 65K10.

Keywords. Norton-Hoff operator, Free interfaces, BV spaces, Rigid bodies, Generalized Schauder fixed-point.

\section{Introduction}

We deal with rigid bodies motion connected to non-smooth evolutions of viscoplastic flows. The fluid's motion is governed by the incompressible quasi-static Norton-Hoff model, whose the coefficients are non-cylindrical, coupled with the time-dependent convection system. The Norton-Hoff operator is inherently nonlinear. The main purpose of this work is to reveal the relationship between a rigid body, established of visco-plastic materials, immersed in visco-plastic flow and a created free interface between two non-miscible flows solutions to the system (2)-(5), (6). The principal results are argued in Theorem 3.2 and 4.1 .

The main difficulties in this analysis are:

1. The coupling between the velocity and the moving domain involves non cylindrical coefficients.

2. The flow's velocity is not regular enough which does not allow to adopt the tools of the classical evolution. 
3. The compactness in time and space is required but we have no estimate on $\partial_{t} u$.

In recent years, many papers concerning the study of rigid body motion in either incompressible or compressible flows governed mainly by the NavierStokes equations have been published in the literature, (see $[4,5,8,13,14]$ ). Temam has proven that velocities describe rigid body motion if and only if their strain tensor vanishes in the classical sense, (see [15]). In this work, we extend the existing formulation to visco-plastic flows, governed by the quasistatic Norton-Hoff model (see $[10,11]$ ), in a weak sense by treating the phenomena of free interfaces in visco-plastic flows.

The contribution of our work enlarges the field of free boundaries and the frame of rigid bodies motion. It has combined results.

1. The first novelty is devoted to provide an existence result of a free interface created by two non-miscible flows of Norton-Hoff type which has never been studied. This result complements and encompasses the Stokes case when the exponent of the material $p$ goes to 2 .

2. The next one is to establish a new variant and extension of Theorem 5.1 in the spirit of the work [15]. It is corroborated that a visco-plastic flow is converted to a rigid body when its viscosity is large enough.

3. The last one is rather implicit. In fact from the previous two points, we are able to deduce an existence result to a mixed system of a rigid body, constituted of visco-plastic materials, immersed in a visco-plastic flow as a limit state of two non-miscible fluids problem when one of the viscosities is large enough. As far as we know, this result does not exist in the literature.

The reminder of this paper is organized as follows. Section 2 recalls some properties connected to the static state. In Sect.3, we first stand the model that describes the problematic. Second, we treat the phenomena of free interfaces between two non-miscible Norton-Hoff fluids in the quasi-static case coupled with the time-dependent convection equations. Then the existence result to the considered problem is argued. It is based on the non-smooth theory of evolution techniques coupled with the generalized Schauder fixed point theorem applied to an established iterative process. Moreover, we prove the weak compactness of the solutions set. The last section is devoted to the formulation of the rigid body motion. We establish that a rigid body motion is reached when the viscosity of the fluid occupying the domain is large enough which recovers the classical formulation in a weak sense.

\section{Preliminaries}

\subsection{Functional setting}

Let us introduce the adequate functional framework in space. Consider a smooth bounded domain $D$.

$\mathcal{W}=W_{0}^{1, p}\left(D, \mathbb{R}^{N}\right)^{3}, \quad \mathcal{W}_{\text {div }}=\{v \in \mathcal{W}$, s.t. $\operatorname{div}(v)=0$ on $D\}, \quad L_{0}^{2}(D)=L^{2}(D) / \mathbb{R}$. 
Further, we introduce the set of the admissible right hand sides,

$$
\mathcal{W}^{\prime}=W^{-1, p^{\prime}}\left(D, \mathbb{R}^{N}\right)^{3},
$$

which is the topological dual space of $\mathcal{W}$ endowed with its natural norm, where $p^{\prime}$ is the algebric dual of $p$. We define $\langle\cdot, \cdot\rangle=\langle\cdot, \cdot\rangle_{V^{*}, V}$ with $V^{*}$ is the topological dual space of $V$. All the other functional spaces used with their natural structure except the mentioned.

The following result provides a Banach space structure for the spaces $\mathcal{W}$ and $\mathcal{W}_{\text {div }}$.

Proposition 2.1. The mapping $\|\cdot\|$ defined from $\mathcal{W}$ to $\mathbb{R}^{+}$by

$$
\|v\|=\left(\int_{D}|\varepsilon(v)|^{p}\right)^{\frac{1}{p}},
$$

is a norm on $\mathcal{W}$ equivalent to the usual one (see $[9,12])$. Where $|\cdot|$ means the Eucledian norm of matrices in $\mathbb{R}^{N^{2}}$.

\subsection{Static case}

We need to recall some properties of the static state.

Definition 2.1. We introduce the Norton-Hoff functional or the so-called compliance functional given by

$$
\begin{aligned}
\Phi_{D} & : \mathcal{W}(\Omega) \longrightarrow \mathbb{R} \\
v & \longrightarrow \int_{D} \frac{K_{c}}{p}|\varepsilon(v)|^{p}-f v .
\end{aligned}
$$

The existence and uniqueness of a solution to the Norton-Hoff problem $\mathcal{P}$ is derived from the properties of $\Phi_{D}$.

Proposition 2.2. The functional $\Phi_{D}$ is strictly convex, weakly l.s.c., Gateaux differentiable and coercive.

The well-posedness of the static Norton-Hoff problem is supplied by the following result. The proof given in [9], is based on the properties of the compliance functional.

Theorem 2.1. The Norton-Hoff mixed problem in the static case has a unique solution $(u, P)$ in $\mathcal{W} \times L_{0}^{2}$.

\section{Free interfaces in visco-plastic flows}

We consider two fluids of Norton-Hoff type with the same exponent $p$ occupying a fixed region $D$ of $\mathbb{R}^{N}, N=2,3$, and undergoing the action of time-dependent volume source $f$. We assume that the mapping of the exterior exertion is sufficiently slow in order to neglect the inert effects. Then the evolution is quasi-static. The fluids are supposed non-miscible and we denote by $\alpha$ and by $\beta$ their consistencies. 


\subsection{Two phases model of Norton-Hoff problem}

The evolution, on a time interval $I=(0, T)$, is a solution of the following non-linear problem of transmission formulated in the cylinder $I \times D$.

$$
\left\{\begin{array}{lll}
K(t, x)|\varepsilon(u)|^{p-2} \varepsilon(u)-P I d=\sigma & \text { in } \quad I \times D, \\
-\operatorname{div}(\sigma)=f & \text { in } I \times D, \\
\operatorname{div}(u)=0 & \text { in } I \times D,
\end{array}\right.
$$

with the homogeneous Dirichlet boundary conditions

$$
u=0 \text { on } I \times \partial D,
$$

and let consider the following initial state

$$
u(0, .)=u_{0}(.) \text { in } D \text {. }
$$

Where $D$ denotes a smooth bounded open subset, locally on one side of its $C^{2}$-boundary $\partial D, \sigma$ is the Cauchy stress tensor, $\varepsilon(u)=\frac{1}{2}\left(\nabla(u)+\nabla(u)^{*}\right)$ is the linearized strain velocity tensor, $\nabla$ is the gradient operator, $p$ is the exponent of the material; $1<p<2$ : it is the sensibility coefficient of the material to the strain velocity tensor, $P$ is the hydrostatic pressure, $I d$ is the identity tensor, $f$ is the density of the gravitation acting on the fluid and $u_{0}$ is a given data. The first equation designates the behavior law, the second describes the balance state and the third one prescribes the incompressibility of the fluid during the evolution. The coefficient $K(t, x)$ is linked to the viscosities of the fluids at the instant $t$ on the position $x$. Let

$$
\begin{aligned}
K(t, x) & =\alpha(t)+\beta(t), \\
\alpha(t) & =\alpha \chi_{\Omega_{t}}, \\
\beta(t) & =\beta \chi_{\Omega_{t}{ }^{c}},
\end{aligned}
$$

where $\Omega$ is a bounded subdomain of $D$ occupied by the fluid of consistency $\alpha$ at the initial time, and, thus $K(t, x)$ is a non-smooth function with respect to the moving domain $\Omega_{t}$ with complementary $\Omega_{t}^{c}$ which makes sense to the existence of the free boundary. Notice that $\Omega=\Omega(t=0)$ is assumed to be locally on one side of its $C^{2}$-boundary $\partial \Omega$.

Remark 3.1. Our point of view is to consider the solution as an evolution of the measurable-domain $\Omega$ and, thus an evolution of the interface between the two non-miscible fluids. We look for a solution to the system (2)-(5) as a fixed point of an established operator via a virtual evolution of the interface with a velocity $u_{V}$. The velocity field $u_{V}$ is the solution of the Norton-Hoff model with respect to a fixed velocity $V$. We may use evolution technicalities since we deal with a transmission problem.

Remark 3.2. The Norton-Hoff model is not regular enough, then we are not able to use the classical theory of evolution in which the flow mapping has to be associated to a vector field whom the space-regularity is, at least, Lipschitzian. Nevertheless, non-smooth evolution will be considered. We will use some new techniques of the speed method for shape analysis that were introduced in [1] and extended in [18]. 
Remark 3.3. Knowing that Sobolev functions cannot have discontinuities along hypersurfaces, as, on the contrary, is required by the model above, the Sobolev space analysis is then no longer appropriate for this kind of problems. For a rigorous presentation, the main tool is the spac BV.

\subsection{Non-smooth evolution of domains}

The space of bounded variations is introduced by

$$
B V(D)=\left\{v \in L^{1}(D) \text { s.t. } \nabla v \in \mathcal{M}^{1}(D)\right\},
$$

where $\mathcal{M}^{1}(D)$ is the Banach space of bounded measures endowed with its natural norm.

We set

$\mathcal{E}^{1,1}=\left\{v \in L^{1}(I, D)^{3}\right.$ s.t. div $v \in L^{1}(I, D)$ and $v \cdot n=0$ in $\left.W^{-1,1}(\partial D)\right\}$,

where $n$ is the outward normal unit vector of $\partial D$.

We denote by

$$
\mathcal{V}=\mathcal{E}^{1,1} \cap\left\{v \in L^{1}\left(I, B V(D)^{3}\right) \text { s.t. } \operatorname{div}(v)_{-}, \operatorname{div}(v)_{+} \in L^{1}\left(I, L^{\infty}(D)\right)\right\},
$$

where $\operatorname{div}(v)_{+}$(respect. $\operatorname{div}(v)_{-}$) is the positive (respect. negative) part of $\operatorname{div}(v)$.

Let

$$
\mathcal{L}=L^{1}\left(I, L^{1}(D)\right) \cap C^{0}\left(I, L^{1}(D)\right) \cap L^{1}(I, B V(D)) .
$$

Thanks to the incompressibility condition div $u=0$ and to the boundary one $u=0$ on $I \times \partial D$ the volume is preserved and the domain $D$ is fixed during the evolution process, which restricts the previous framework to the following set

$\mathcal{V}_{0}=\left\{v \in L^{1}(I, D)^{3}\right.$ s.t. $\operatorname{div} v=0$, and $u=0$ in $\left.W^{-1 / 2, p}(\partial D)\right\} \cap L^{1}\left(I, B V(D)^{3}\right)$,

By referring to $[1,18]$ we have the following result.

Theorem 3.1. For all $V$ in $\mathcal{V}$ and measurable subset $\Omega$ of $D$, there exists a unique solution $\chi$ in $\mathcal{L}$ to the convection problem

$$
\begin{cases}\dot{\chi}(t)+\nabla \chi(t) \cdot V=0 & \text { in } D, \text { a.e. } t \in I, \\ \chi(0)=\chi_{\Omega} & \text { in } D,\end{cases}
$$

and for almost every $t$ in $I, \chi(t)$ is a characteristic function.

Moreover,

$$
\text { if }\left(V_{n}\right)_{n \in \mathbb{N}} \rightarrow V \text { in } \mathcal{V} \text { then }\left(\chi^{V_{n}}\right)_{n \in \mathbb{N}} \rightarrow \chi^{V} \text { in } \mathcal{L} .
$$

Besides, the convergence of the characteristic sequence $\left(\chi^{V_{n}}\right)_{n \in \mathbb{N}}$ is weak-star in the space $L^{\infty}(I, D)$.

Remark 3.4. The weak flow does not affect the domain point by point as does the classical evolution, but it affects the domain's characteristic.

In order to establish the link between rigid bodies motion and visco-plastic fluids, we have to argue an existence result of the free interface. We start by supplying an iterative process describing the evolution of the system (2)(5), (6) which allows us to exhibit the so-called generalization of the Schauder operator defined on a particular convex subset of a Banach-space (see $[16,17]$ ). 


\subsection{Process}

Let $V^{n}$ be a given velocities sequence, then the weak flow provides the associated characteristic function of the convected domains $\Omega_{t}\left(V^{n}\right)$, hence the viscosity is given by $K_{\Omega_{t}\left(V^{n}\right)}=K^{n+1}$. While, by resolving the Norton-Hoff equations we recover the fluid's velocity $u_{V^{n}}=u^{n+1}$. Thus, the process continues.

$$
V^{n} \text { given } \stackrel{\text { weakflow }}{\longrightarrow} K_{\Omega_{t}\left(V^{n}\right)}=K^{n+1} \stackrel{\text { Norton--Hoff }}{\longrightarrow} u_{V^{n}}=u^{n+1} .
$$

The velocity $u^{n+1}$ fulfills the hereafter system with the same boundary and initial conditions (3), (4).

$$
\mathcal{P}\left(V^{n}\right) \begin{cases}-\operatorname{div}\left(K^{n+1}\left|\varepsilon\left(u^{n+1}\right)\right|^{p-2} \varepsilon\left(u^{n+1}\right)-P I d\right)=f & \text { in } I \times D, \\ \operatorname{div} u^{n+1}=0 & \text { in } \quad I \times D .\end{cases}
$$

We state here the first main result of this work.

Theorem 3.2. The two phases Norton-Hoff problem (2)-(5), (6), has at least, a solution in $\mathcal{V}_{0}$ : there exists a virtual velocity $V$ such that

$$
u_{V}=V
$$

Remark 3.5. The last important result provides in a weak sense the existence of the free interface between the two fluids and ensure that the flows are kept non-miscible in the evolution process.

\subsection{The generalized Schauder operator}

In the sequel, we denote $\mathcal{O}_{\text {mes }}$ the set of measurable domains included in $D$. From the previous process, one can extend the following mapping

$$
\mathcal{G}: \quad V \longrightarrow u_{V}
$$

which takes value into the closed, bounded, and convex ball $\mathcal{B}_{u}$ included in $\mathcal{V}_{0}$, whose the radius is given subsequently. The velocity $u_{V}$ fulfills the system $\mathcal{P}(V)$ with the same boundary and initial conditions (3), (4).

The generalized Schauder fixed-point, (see, [17]), will depend on the continuity and the compactness of the extended mapping $\mathcal{G}$ in a weak sense. Unless the boundedness of $\partial_{t} u$ does not exist which makes the compactness in time and in space more difficult. In fact, $\partial_{t} u$ describes the shape derivative of the state $u$ and such a derivative needs more regularity then our case (see [18]). The main key of the proof is the use of the non-smooth evolution techniques.

3.4.1. Weak continuity result. We recall here several basic definitions and concepts.

Definition 3.1. Let $E$ be a Banach space and $X \subset E$ be a nonempty set. An operator $T: X \rightarrow E$ is said to be sequentially weakly continuous on the set $X$ if for every sequence $x_{n}$ in $X$ and $x$ in $X$ such that $x_{n} \rightarrow x$ we have that $T x_{n} \rightarrow T x$. 
Definition 3.2. Due to the Eberlin-Smulian's Theorem ([6], Theorem 8.12.4), it is well known that if a set $K$ is weak compact, then each sequentially weakly continuous mapping $T: K \rightarrow E$ is weakly continuous.

Therefore, it may be possible to look for a fixed point in the weak topology setting. As a tool to this intention, we rely on the version of Schauder principle which was obtained by Arino et al. [2].

Proposition 3.1. Let $\left(\Omega_{n}\right)_{n \in \mathbb{N}}$ be a sequence in $\mathcal{O}_{\text {mes }}$ such that the characteristic function $\left(\chi_{\Omega_{n}}=\chi_{n}\right)_{n \in \mathbb{N}}$ converges strongly towards a characteristic function $\chi_{*}=\chi_{\Omega_{*}}$, with $\Omega_{*} \in \mathcal{O}_{\text {mes }}$, in $L^{r}\left(I, L^{q}(D)\right)$ for all $1 \leq q, r<\infty$ (it is equivalent to: $V^{n} \rightarrow V^{*}$ in $\left.\mathcal{V}_{0}\right)$. Then $\left(u_{\Omega_{n}}\right)_{n \in \mathbb{N}}$ solution of $\mathcal{P}\left(V^{n}\right)$ converges weakly in $\mathcal{W}_{\text {div }}$ towards $u_{\Omega_{*}}$ solution of $\mathcal{P}\left(V^{*}\right)$.

In order to argue the proof, the following lemmas are crucial.

Lemma 3.1. Let $\left(y_{n}\right)_{n \in \mathbb{N}}$ be a sequence that converges weakly to $y_{1}$ in $L^{p}(I \times$ $\mathcal{W})$, for all $1 \leq p \leq 2$. Moreover, assume that for a.e. $t \in I,\left(y_{n}\right)_{n \in \mathbb{N}}(t)$ converges weakly to $y_{2}(t)$ in $\mathcal{W}$. Then, for a.e. $t \in I, y_{1}(t)=y_{2}(t)$ in $L^{p}(D)$.

The proof is based on the Banach-Zucks lemma, (see [7]).

Lemma 3.2. There exists a constant $c$ such that if $u$ is the solution of the Norton-Hoff problem then

$$
\|u\|_{L^{2}(I ; \mathcal{W})} \leq \frac{c_{P}}{\alpha}\|f\|_{L^{\infty}\left(I ; L^{p^{\prime}}(D)\right)}^{\frac{1}{p-1}},
$$

where $c_{P}$ is the Poincare's constant and $\alpha$ is the consistency of the fluid occupying the set $\Omega$. Let $\frac{c_{P}}{\alpha}\|f\|_{L^{\infty}\left(I ; L^{p^{\prime}}(D)\right)}^{\frac{1}{p-1}}$ be the radius of the above mentioned ball $\mathcal{B}_{u}$.

Proof. It is easy to derive

$$
\int_{D} K|\varepsilon(u)|^{p} \leq\|f\|_{L^{p^{\prime}}(D)}\|u\|_{L^{p}(D)},
$$

else Poincare's inequality provides

$$
\alpha\|u\|_{\mathcal{W}} \leq c_{P}\|f\|_{L^{p^{\prime}}(D)}^{\frac{1}{p-1}}, \quad \forall t \in I
$$

then

$$
\|u\|_{\mathcal{W}} \leq \frac{c_{P}}{\alpha}\|f\|_{L^{\infty}\left(I ; L^{p^{\prime}}(D)\right)}^{\frac{1}{p-1}}, \quad \forall t \in I
$$

Moreover,

$$
\|u\|_{L^{\infty}(I ; \mathcal{W})} \leq \frac{c_{P}}{\alpha}\|f\|_{L^{\infty}\left(I ; L^{p^{\prime}}(D)\right)}^{\frac{1}{p-1}} .
$$

As $L^{\infty}$ is continuously embedded in $L^{2}$, it follows

$$
\|u\|_{L^{2}(I ; \mathcal{W})} \leq \frac{c_{P}}{\alpha}\|f\|_{L^{\infty}\left(I ; L^{p^{\prime}}(D)\right)}^{\frac{1}{p-1}} .
$$


Lemma 3.3. One can easily deduce that there exists a constant $c>0$ such that

$$
\int_{D} \sigma(u) \cdot \varepsilon(u) \leq c\|f\|_{L^{\infty}\left(I ; L^{p^{\prime}}(D)\right)}^{\frac{p}{p-1}} .
$$

We denote by $\left(u_{n}\right)_{n \in \mathbb{N}}=\left(u_{\Omega_{n}}\right)_{n \in \mathbb{N}}$ and $\left(\chi_{n}\right)_{n \in \mathbb{N}}=\left(\chi_{\Omega_{n}}\right)_{n \in \mathbb{N}}$.

The following lemma shows how to overcome the lack of compactness in time and in space in order to obtain the proof of Theorem 3.2.

Lemma 3.4. The sequence $\left(u_{n}\right)_{n \in \mathbb{N}}$, with respect to a subsequence, is strongly convergent to $u_{*}$ in the $L^{p}(I \times D)$ sense.

Proof. On the one hand, it is already known that there exists a constant $c$ such that

$$
\left\|u_{n}\right\|_{L^{p}(I, \mathcal{W})} \leq c
$$

then one can extract a subsequence $\left(u_{n_{k}}\right)_{k \in \mathbb{N}}$ that converges weakly to $u_{*}$ in $L^{p}(I ; \mathcal{W})$. On the other hand, using the $L^{\infty}$-estimate $\left\|u_{n}\right\|_{L^{\infty}(I ; \mathcal{W})} \leq c$, then $\left\|u_{n_{k}}\right\|_{L^{\infty}(I ; \mathcal{W})} \leq c$. So, there exists a subsequence $\left(u_{n_{k_{q}}}\right)_{q \in \mathbb{N}}$ that converges weakly-star to $w$ in $L^{\infty}(I ; \mathcal{W})$. It transpires that for all $\varphi$ in the topological dual space $L^{1}\left(I ; \mathcal{W}^{\prime}\right)$, we have

$$
\lim _{q \uparrow \infty} \int_{0}^{T}\left\langle u_{n_{k_{q}}}, \varphi\right\rangle d t=\int_{0}^{T}\langle w, \varphi\rangle d t .
$$

Hence, by separability of the space $L^{1}$, the sequence $\left\langle u_{n_{k_{q}}}, \varphi\right\rangle_{q \in \mathbb{N}}$ converges strongly to $\langle w, \varphi\rangle$ in $L^{1}(0, T)$. Then there exists a subsequence denoted also by $\left(u_{n_{k_{q}}}\right)_{q \in \mathbb{N}}$ such that $\left\langle u_{n_{k_{q}}}, \varphi\right\rangle_{q \in \mathbb{N}}(t)$ converges to $\langle w, \varphi\rangle(t)$ a.e. $t$ in $(0, T)$. It comes that

$$
\text { a.e. } t \text { in }(0, T) ;\left(u_{n_{k_{q}}}\right)_{q \in \mathbb{N}}(t) \rightarrow w(t) \text { in } \mathcal{W} \text {. }
$$

It follows that a.e. $t$ in $(0, T),\left(u_{n_{k_{q}}}\right)_{q \in \mathbb{N}}(t)$ converges strongly to $w(t)$ in $L^{p}(D)$. Accordingly, Lemma 3.1 supplies that $w(t)=u_{*}(t)$. Moreover, $w(t)$ is unique, so the whole $\left(u_{n_{k}}\right)_{k \in \mathbb{N}}$ converges strongly to $u_{*}(t)$ in $L^{p}(D)$.

Set

$$
g_{k}(t)=\left\|u_{n_{k}}(t)-u_{*}(t)\right\|_{L^{p}(D)},
$$

then for a. e. $t \in(0, T), \lim _{k \uparrow \infty} g_{k}(t)=0$ and $\left|g_{k}(t)\right| \leq c$, where $c$ belongs to $L^{1}(0, T)$. Thus, the Lebegue's Theorem argues that

$$
\lim _{k \uparrow \infty} g_{k}=0, \text { in } L^{1}(0, T),
$$

which provides the claim.

Lemma 3.5. From Theorem 3.1 and Lemma 3.4, the corresponding characteristics sequence $\left(\chi_{n}\right)_{n \in \mathbb{N}}(t)$ is strongly convergent to $\chi_{*}(t)=\chi_{\Omega_{*}}(t)$ in the $L^{p^{\prime}}(D)$ sense. 
Proof. Green's formula applied to the convection system provides that for all $\varphi$ in $C^{1}\left(I, C_{0}^{\infty}(D)\right)$ with $\varphi(T)=0$,

$$
\int_{I \times D} \chi_{n}\left(-\partial \varphi+u_{n} \cdot \nabla \varphi\right) d x d t+\int_{D} \chi_{0} \varphi(0) d x=0 .
$$

As $\chi_{n}$ converges star-weak to $\xi$ in $L^{\infty}(I \times D)$ and $u_{n}$ converges strongly to $u_{*}$ in $L^{1}(I \times D)$, then the couple $\left(\xi, u_{*}\right)$ verifies the convection system $(6)$. With the fact that $u_{*}$ belongs to $\mathcal{V}_{0}$, from Theorem 3.1 one can deduce that the limit $\xi$ is unique and it is a characteristic function, which means that $\xi(t)=\chi_{\Omega}(t)$. Moreover, by using a classical lemma we prove that

$$
\left(\chi_{n}\right)_{n \in \mathbb{N}} \rightarrow \chi, \text { in } L^{q}(I \times D), \forall q \geq 1 .
$$

Lemma 3.6. The vector $u_{*}$ is a solution to $\mathcal{P}\left(u_{*}\right)$. Moreover, $u_{*}=u_{V_{*}}$.

Proof. The Norton-Hoff problem is variational. It is easy to check the following inequality, for any $n \in \mathbb{N}$ :

$\int_{D} \frac{K_{n}}{p}\left|\varepsilon\left(u_{n}\right)\right|^{p}-\int_{D} f u_{n} \leq \int_{D} \frac{K_{n}}{p}|\varepsilon(v)|^{p}-\int_{D} f v=\Phi_{n}(v), \quad \forall v \in \mathcal{W}_{d i v}$, where $\Phi_{n}$ is a convex and a Gateaux differentiable mapping as mentioned previously.

As the sequence $\left(K_{n}\right)_{n \in \mathbb{N}}$ converges strongly towards $K_{*}$ in $L^{q}(D)$ for all $q \geq 1$, then it yields

$\lim \inf _{n \uparrow \infty}\left\{\int_{D} \frac{K_{n}}{p}\left|\varepsilon\left(u_{n}\right)\right|^{p}-\int_{D} f u_{n}\right\} \leq \int_{D} \frac{K_{*}}{p}|\varepsilon(v)|^{p}-\int_{D} f v, \quad \forall v \in \mathcal{W}_{\text {div }}$,

then

$\lim \inf _{n \uparrow \infty} \int_{D} \frac{K_{n}}{p}\left|\varepsilon\left(u_{n}\right)\right|^{p}+\lim \inf _{n \uparrow \infty}\left(-\int_{D} f u_{n}\right) \leq \int_{D} \frac{K_{*}}{p}|\varepsilon(v)|^{p}-\int_{D} f v, \quad \forall v \in \mathcal{W}_{d i v}$, which involves

$\lim \inf _{n \uparrow \infty}\left(\int_{D} \frac{K_{n}}{p}\left|\varepsilon\left(u_{n}\right)\right|^{p}\right)-\int_{D} f u_{*} \leq \int_{D} \frac{K_{*}}{p}|\varepsilon(v)|^{p}-\int_{D} f v, \quad \forall v \in \mathcal{W}_{\text {div }}$.

It arises that

$$
\int_{D} \frac{K_{n}}{p}\left|\varepsilon\left(u_{n}\right)\right|^{p}=\left\|\left(\frac{K_{n}}{p}\right)^{1 / p} \varepsilon\left(u_{n}\right)\right\|_{L^{p}}^{p}
$$

whereas, the mapping $v \longrightarrow\left\|\left(\frac{K_{n}}{p}\right)^{1 / p} \varepsilon(v)\right\|_{L^{p}}$ is weakly l.s.c., convex onto $\mathcal{W}_{\text {div }}$.

Indeed, on the one hand, the function $\frac{K_{n}}{p}\left|\varepsilon\left(u_{n}\right)\right|^{p}$ is positive and $\sup _{n} \int_{D}$ $\frac{K_{n}}{p}\left|\varepsilon\left(u_{n}\right)\right|^{p}<\infty$, on the other hand,

$$
\left(\frac{K_{n}}{p}\right)^{1 / p} \varepsilon\left(u_{n}\right) \rightarrow\left(\frac{K_{*}}{p}\right)^{1 / p} \varepsilon\left(u_{*}\right), \quad n \uparrow \infty \text { in } L^{1}(D) .
$$


Whence, by using Fatou's lemma (see [3]), we get

$$
\int_{D} \frac{K_{*}}{p}\left|\varepsilon\left(u_{*}\right)\right|^{p} \leq \lim \inf _{n \uparrow \infty} \int_{D} \frac{K_{n}}{p}\left|\varepsilon\left(u_{n}\right)\right|^{p} .
$$

It follows that

$\int_{D} \frac{K_{*}}{p}\left|\varepsilon\left(u_{*}\right)\right|^{p}-\int_{D} f u_{*} \leq \int_{D} \frac{K_{*}}{p}|\varepsilon(v)|^{p}-\int_{D} f v=\Phi_{*}(v), \quad \forall v \in \mathcal{W}_{d i v}$.

Thus, $u_{*}$ realizes the minimum of $\Phi_{*}$. By uniqueness $u_{*}=u_{\Omega_{*}}$, and so the proof is done.

Proof of Proposition 3.1. Lemmas 3.4, 3.5, 3.6 confirm that the operator $\mathcal{G}$ is sequentially weakly continuous on $\mathcal{B}_{u}$. With the fact that the ball $\mathcal{B}_{u}$ of the reflexive Banach space $\mathcal{W}$ is weakly compact and by the use of Definition 3.2 we get that the operator $\mathcal{G}$ is weakly continuous.

3.4.2. Weak compactness result. We prove that the set $\mathcal{G}(\mathcal{B})$ is weakly relatively-compact in $\mathcal{V}_{0}$.

Proposition 3.2. For any integer $n \in \mathbb{N}$ we denote $\left(V_{n+1}\right)_{n \in \mathbb{N}}=\mathcal{G}\left(\left(V_{n}\right)_{n \in \mathbb{N}}\right)$ which is a sequence of $\mathcal{G}(\mathcal{B}) \subset \mathcal{V}_{0}$, then one can extract a subsequence $\left(V_{n_{k}}\right)_{k \in \mathbb{N}}$ that converges weakly in $\mathcal{G}(\mathcal{B})$.

Proof. We can notice that $\left(V_{n+1}\right)_{n \in \mathbb{N}}: t \longrightarrow\left(u_{\Omega_{t}\left(V_{n}\right)}\right)_{n \in \mathbb{N}}$. According to lemma 3.2 , it transpires that there exists a constant $c$ independent on $n$ such that

$$
\left\|V_{n+1}\right\|_{\mathcal{W}_{\text {div }}} \leq c .
$$

The compactness argument yields to an extracted subsequence $\left(V_{n_{k}}\right)_{k \in \mathbb{N}}$ which converges weakly to $V_{*}$ in $\mathcal{V}_{0}$. On the one hand, we result by Lemma 3.5 that $\left(\chi_{\Omega_{t}\left(V_{n_{k}}\right)}\right)_{k \in \mathbb{N}}$ converges in $L^{r}\left(I, L^{q}(D)\right)$ strongly towards the characteristic function $\chi_{*}=\chi_{\Omega_{t}\left(V_{*}\right)}$. On the other hand, Proposition 3.1 involves that $\left(u_{\Omega_{t}\left(V_{n_{k}}\right)}\right)_{k \in \mathbb{N}}$ converges weakly towards $\left(u_{\Omega_{t}\left(V_{*}\right)}\right)$ in $\mathcal{B} \subset \mathcal{V}_{0}$ and accordingly $\left(V_{n_{k}}\right)_{k \in \mathbb{N}}$ converges weakly to $V_{*}$. Whereas

$$
V_{*}: t \longrightarrow u_{\Omega_{t}\left(V_{*}\right)} \text {. }
$$

Furthermore, the weak continuity of the operator $\mathcal{G}$ yields that $V_{*}$ belongs to $\mathcal{G}(\mathcal{B})$. Whence, the weak compactness result of the operator $\mathcal{G}$ is supplied.

We are now ready to prove the first main result of this article.

Proof of Theorem 3.2. We shall employ the generalized Schauder fixed point result which has been established in [2]. The ball $\mathcal{B}_{u}$ is weakly compact convex subset of the reflexive Banach space $\mathcal{W}$. Then, the weakly continuous operator $\mathcal{G}: \mathcal{B}_{u} \rightarrow \mathcal{B}_{u}$ has a fixed point. Which involves a weak existence result of the free interface.

Theorem 3.2 encompasses the result that were obtained in the Stokes state. 


\subsubsection{Compactness of the solutions set.}

Theorem 3.3. Let $S$ be the solutions set of the diphasic Norton-Hoff problem.

$$
S=\left\{(\chi, u) \in \mathcal{L} \times \mathcal{V}_{0} ; \text { s.t. }(\chi, u) \text { solution of }(2)-(5),(6)\right\} .
$$

Then the set $S$ is compact for the weak topology induced by $\mathcal{L} \times \mathcal{V}_{0}$.

Proof. We may use the same setting as before in order to get the proof. Let $\left(u_{n}\right)_{n \in \mathbb{N}}$ be a sequence that belongs to $S$. Then, under the Lemma 3.2 given above, the sequence $\left(u_{n}\right)_{n \in \mathbb{N}}$ is bounded in the closed bounded ball $\mathcal{B} \subset \mathcal{V}_{0}$. Therefore, on one side, by compactness argument one can extract a subsequence, also denoted $\left(u_{n}\right)_{n \in \mathbb{N}}$, which converges weakly towards $u_{*}$ in $\mathcal{V}_{0}$. On the other side, by Lemma 3.5 we deduce that $\chi_{\Omega_{t}\left(V_{n}\right)}$ converges strongly in $L^{r}\left(I, L^{q}(D)\right)$ towards the characteristic function $\chi_{*}=\chi_{\Omega_{t}\left(u_{*}\right)}$ solution to $(6)$. Moreover, Lemmas 3.4, 3.6 provide that $u_{*}$ is a solution of the problem $\mathcal{P}$. Accordingly, $\left(\chi_{*}, u_{*}\right)$ belongs to $S$. Hence, $S$ is a weak compact subset of $\mathcal{V}_{0}$. Thus, the set of all possible interfaces is weakly compact with respect to the induced topology.

\section{Rigid body}

We focus on the fluid of consistency $\alpha$ occupying the measurable domain $\Omega(t)$ $\subset D$. Let $Q=\cup_{0<t<T}\{t\} \times \Omega(t)$ be the tube built during the evolution process. The classical characterization of rigid bodies is based on regular enough velocity, (see [15], Theorem 5.1 on p. 76). The main aim of this section is to provide a weak formulation of the rigid bodies established of visco-plastic flows modeled by the Norton-Hoff operator.

Theorem 4.1. Let $\left(u(t, x), \chi_{\Omega(t)}(x)\right)$ be a solution of the system (2)-(5), (6) and let $\alpha$ be the viscosity of the flow occupying the set $\Omega(t)$ throughout the evolution process. If $\alpha$ is large enough, then

the tube $Q$ is a rigid body if and only if $\varepsilon(u(t))=0$ a. e. in the $L^{p}(Q)-$ sense.

The following results are crucial in order to provide the proof of the Theorem 4.1.

Lemma 4.1. Let $\beta>0$ and assume that $\left(\alpha_{n}\right)_{n \in \mathbb{N}}=\left(\frac{1}{\nu_{n}}\right)_{n \in \mathbb{N}}$ with $\nu_{n} \longrightarrow 0$ when $n \uparrow \infty$. Then, according to Theorem 3.2 , there exists a couple $\left(u^{n}, \chi^{n}\right)_{n \in \mathbb{N}}$ that belongs to $\mathcal{V}_{0} \times \mathcal{L}$ and satisfies the system of Eqs. (2)-(5), (6).

Lemma 4.2. From the system (2)-(5), (6) itself we get the following uniform estimate: there exists a positive constant $C$ such that

$$
\text { a. e.t in }(0, T), \int_{\Omega_{t}}\left|\varepsilon\left(u^{n}(t)\right)\right|^{p} d x \leq \nu_{n} C \text {. }
$$

Proposition 4.1. By the same techniques used in the proof of Propositions 3.1, 3.2, we get the existence of a limit couple $(u, \chi)$ of the sequence $\left(u^{n}, \chi^{n}\right)_{n \in \mathbb{N}}$ that satisfies the system (2)-(5), (6). 
Lemma 4.3. We have the following equality

$$
\text { a. e. } \operatorname{tin}(0, T), \quad \chi_{\Omega}(t) \varepsilon(u(t))=0 \quad \text { in } Q \text {. }
$$

Proof. We set $\chi_{n}=\chi_{\Omega_{n}}$, then Lemma 4.2 implies that

a.e. $t$ in $(0, T), \chi_{n}(t)\left|\varepsilon\left(u^{n}(t)\right)\right| \longrightarrow 0$, in the $L^{p}(D)-$ sense,

whereas,

a. e. $t$ in $(0, T), \varepsilon\left(u^{n}(t)\right) \rightarrow \varepsilon(u(t)) \quad$ in the $L^{p}(D)-$ sense,

and

a. e. $t$ in $(0, T), \chi_{n}(t) \longrightarrow \chi(t)$, in the $L^{p}(D)-$ sense for all $p \geq 1$,

therefore,

a. e. $t$ in $(0, T), \chi_{n}(t) \varepsilon\left(u^{n}(t)\right) \rightarrow \chi(t) \varepsilon(u(t))$, in the $L^{1}(D)-$ sense.

Hence, for all $\psi$ in $\mathcal{D}$, the set of $C^{\infty}$ - matricesfunctions, we obtain

$\int_{D} \chi_{n}(t) \varepsilon\left(u^{n}(t)\right) \psi d x=\int_{D} \chi(t) \psi \varepsilon\left(u^{n}(t)\right) d x+\int_{D}\left(\chi_{n}(t)-\chi(t)\right) \psi \varepsilon\left(u^{n}(t)\right) d x$.

On the one side, by passing to the limit when $n \uparrow \infty$ and via Cauchy-Schwartz inequality, we get for all $\psi$ in $\mathcal{D}$

$$
\lim _{n \downarrow 0} \int_{D} \chi_{n}(t) \varepsilon\left(u^{n}(t)\right) \psi d x=\int_{D} \chi(t) \varepsilon(u(t)) \psi d x .
$$

On the other side, since $\chi_{n}(t) \mid \varepsilon\left(u^{n}(t) \mid \longrightarrow 0\right.$ in the $L^{p}(D)$-sense, we deduce that

$$
\text { a. e. } t \text { in }(0, T), \chi(t) \varepsilon(u(t))=0, \text { in } L^{p}(D)-\text { sense. }
$$

Thus,

$$
\text { a.e.t in }(0, T), \varepsilon(u(t))=0, \text { in } L^{p}(Q)-\text { sense, }
$$

which gives over to the proof.

In the end, Proposition 4.1 and Lemma 4.3 supply the proof of Theorem 4.1.

Theorem 4.1 hides an interesting corroboration. It provides an existence result to a mixed system of a rigid body, constituted of visco-plastic materials, immersed in a visco-plastic flow as a limit state of two non-miscible fluids problem when one of the viscosities is large enough.

\section{Conclusion}

We have studied the connection between rigid bodies motion established of visco-plastic materials and free interfaces created by two non-miscible visco-plastic flows. We have argued a weak existence result to the considered interfaces and have shown that the set of solutions is compact in a weak sense. We were faced to the fact that the velocity field of the flow, governing the evolution of the interface, is less-regular than classical cases. Nevertheless, we were able to overcome this difficulty by using a coupling between the generalized Schauder 
fixed-point and the theory of non-smooth evolution which enabled us to control the domain's characteristic and consequently the created interfaces. We have supplied a weak formulation of rigid bodies constituted of visco-plastic materials. The question of uniqueness is still open. The results that were obtained encourage further analysis of the proposed techniques including identification of free interfaces in the thermal case.

\section{Acknowledgments}

Supported by the funds of the project number 1432-18.

\section{References}

[1] Ambrosio, L.: Mathematicals aspects of evolving interfaces. Lecture Note in Mathematics. Springer, Berlin (2003)

[2] Arino, O, Gautier, S, Penot, J.P.: A fixed point theorem for sequentially continuous mappings with application to ordinary differential equations. Funkcial. Ekvac. 27(3), 273-279 (1984)

[3] Brezis, H.: Analyse fonctionnelle. Théorie et applications. Collection Mathé matiques Appliquées (1983)

[4] Desjardins, B, Esteban, M.J.: Existence of weak solutions for the motion of rigid bodies in a viscous fluid. Arch. Ration. Mech. Anal. 146, 59-71 (1999)

[5] Desjardins, B, Esteban, M.J.: On weak solutions for fluid-rigid structure interaction: compressible and incompressible models. Comm. Partial Differ. Equ. 25, 1399-1413 (2000)

[6] Edwards, R.E.: Functioal Analysis, Theory and Applications. Holt, Rinehart and Winston, New York (1965)

[7] Ekeland, E., Temam, R.: Analyse convexe et problèmes variationnels. Etudes Mathématiques (1974)

[8] Feireisl, E: On the motion of rigid bodies in a viscous fluid. Appl. Math. 47, 463$484(2002)$

[9] Ferchichi, J., Gaied, I.: Well-posedness of the non-linear Norton-Hoff operator via the min-max theory applied to the compliance functional. ISRN Appl. J. 1-9 (2011)

[10] Ferchichi, J, Zolésio, J.P.: Identification of a free boundary in Norton-Hoff flows with thermal effects. J. Comput. Appl. Math. 220(1-2), 181-197 (2008)

[11] Ferchichi, J, Zolésio, J.P.: Study of the Norton-Hoff operator coupled with the evolutive heat equation. J. Math. Anal. Appl. 334, 97-113 (2007)

[12] Geymonat, G, Suquet, P: Functional spaces for Norton-Hoff materials. Math. Meth. Appl. Sci. 8, 206-222 (1986) 
[13] Gunzburger, M.D., Lee, H.C., Seregin, G.A.: Global existence of weak solutions for viscous incompressible flows around a moving rigid body in three dimensions. J. Math. Fluid Mech. 2, 219-266 (1986)

[14] San Martin, J., Starovoitov, V., Tucsnak, M.: Global weak solutions for the two dimensional motion of several rigid bodies in an incompressible viscous fluid. Arch. Ration. Mech. Anal. 161, 113-222 (2002)

[15] Temam, R: Mathematical modeling in continuum mechanics. Arch. Mech. Anal. 95(2), 137-183 (1986)

[16] Xiang, T, Yuan, R.: Krasnoselskii-type fixed point theorems under weak topology setting and applications. Electron. J. Differ. Equ. 35, 1-15 (2010)

[17] Zeidler, E.: Nonlinear functional analysis and its applications. In: Tome, I. (ed.) Fixed Point Theorms. Springer, Berlin (1986)

[18] Zolésio, J.P.: Control of moving domains, shape stabilization and variational tube formulations. Int. Nat. Ser. Num. Math. 155, 329-382 (2007)

J. Ferchichi

Research Unity: ACPDE

University of Monastir

Monastir

Tunisia

e-mail: jamel.ferchichi@fsm.rnu.tn

J. Ferchichi

King Khaled university

Abha

KSA

J. P. Zolésio

OPALE-INRIA Sophia-Antipolis and CNRS

Sophia-Antipolis

France

e-mail: jean-paul.zolesio@sophia.inria.fr

Received: 23 October 2011.

Accepted: 1 September 2012. 